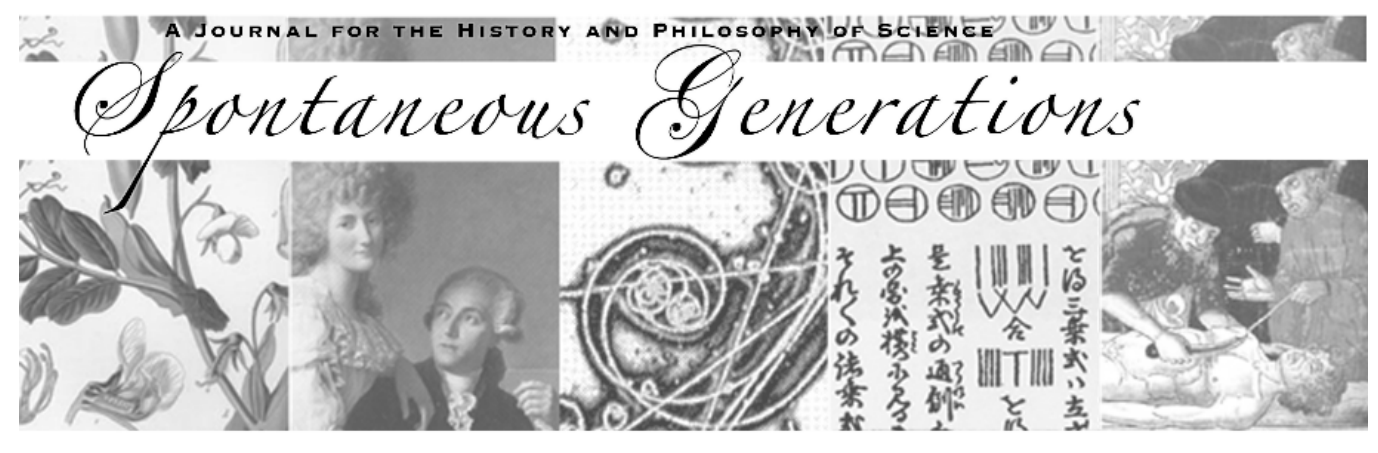

\title{
Visual Representations of Structure and the Dynamics of Scientific
} Modeling

Author(s): William Goodwin

Source: Spontaneous Generations: A Journal for the History and Philosophy of Science, Vol. 6, No. 1 (2012) 131-141.

Published by: The University of Toronto

DOI: $10.4245 /$ sponge.v6i1.17155

\section{EDITORIAL OFFICES}

Institute for the History and Philosophy of Science and Technology

Room 316 Victoria College, 91 Charles Street West

Toronto, Ontario, Canada M5S 1K7

hapsat.society@utoronto.ca

Published online at jps.library.utoronto.ca/index.php/SpontaneousGenerations

ISSN 19130465

Founded in 2006, Spontaneous Generations is an online academic journal published by graduate students at the Institute for the History and Philosophy of Science and Technology, University of Toronto. There is no subscription or membership fee. Spontaneous Generations provides immediate open access to its content on the principle that making research freely available to the public supports a greater global exchange of knowledge. 


\title{
Visual Representations of Structure and the Dynamics of Scientific Modeling*
}

\author{
William Goodwin ${ }^{\dagger}$
}

\begin{abstract}
Understanding what is distinctive about the role of models in science requires characterizing broad patterns in how these models evolve in the face of experimental results. That is, we must examine not just model statics-how the model relates to theory, or represents the world, at some point in time-but also model dynamics-how the model both generates new experimental results and is modified in response to them.

Visual representations of structure play a central role in the theoretical reasoning of organic chemists. Not surprisingly, these representations have changed in important ways-in response to experimental and theoretical developments- throughout the history of organic chemistry. In many cases it is appropriate to understand the visual representations used by organic chemists as models. The evolution of the structural representations of organic chemists therefore provides a clear example of the dynamics of scientific modeling. In this paper I use a conception of scientific modeling drawn from the work of Mary Hesse to examine how the concept of a molecular conformation was incorporated into the visual representations of structure used by organic chemists.
\end{abstract}

\section{INTRODUCTION}

Modern organic chemistry is in large measure the fruit of an idea developed in the second half of the nineteenth century. The idea was, roughly, to employ a visual symbolic system to represent the elemental, and perhaps the atomic, composition and structure of chemical compounds, and then to use that structure to explain the chemical (and some of the physical) properties of the represented compound. This was the germ of a research program that has endured to this day. One remarkable feature of this research program is that it has continued to evolve and has been remarkably fruitful, in spite of the fact that the idea and representations at its core-those of chemical structure-have changed dramatically over the course of its development. Chemists' commitment to a structural approach is much firmer than their commitment to particular conceptions or representations of that structure. Another way to put this might be to say that chemical structure is dynamic; this

* Received 30 April 2012.

$\dagger$ William Goodwin is a philosopher of science affiliated with the University of South Florida. 
has been crucial to the continued success of the research program built around it.

James Conant used to advocate for a historical approach to understanding science on the grounds that only this approach would reveal the "dynamic quality of science" (Conant 1947, 24). Science, for Conant, was "that portion of accumulative knowledge in which new concepts are continuously developing from experiment and observation and lead to further experiment and observation" (Conant 1947, 98). An appreciation for this constructive feedback between experiment and theory was for him essential to "understanding science." Furthermore, it was this dynamic aspect of science that was most likely to be frozen out in philosophical analysis. Philosophers, Conant (and others) noticed, had-and sometimes still have-a tendency to treat the theoretical products of scientists as "museum pieces" frozen in time, rather than as concepts, like chemical structure, which are "always being extended and modified to account for new phenomena" (Hesse 1966, 4).

One of the products of Conant's historical approach has been an increasing appreciation of the role of models and modeling in science. Models, many philosophers have come to realize, are crucial to the dynamics of science. In order to understand how scientists extend their approaches into new territory, it is not sufficient to focus on the abstract laws or theories germane to the field. Instead, much of the relevant cognitive content of science can be captured only in the models that are used to apply the theory in more concrete, particular circumstances. Similarly, when anomalies show up, it is often the models, not the abstract theory, that are reinterpreted or adjusted to accommodate the unexpected results. Appreciating the dynamic character of science and the distinctive role of models in science should therefore include characterizing broad patterns in how these models both lead to, and evolve in the face of, experimental results. That is, we must examine not just model statics-how the model relates to theory, or represents the world, at some point in time-but also model dynamics-how the model both generates new experimental results and is modified in response to them.

One of the important features of the visual representations of structure used by chemists is, or so I have argued elsewhere (Goodwin 2008; 2009), that they are not merely symbols whose cognitive content can be cashed out according to the rules of some formal system. They do have such formal content, but they are not exhausted by it. Instead, they also function as models of the compounds that they are used to represent. In their role as models, these visual representations have been crucial to the dynamics of chemical structure. The visual representations of scientists can play, therefore, an additional, underappreciated role in the dynamics of science by acting as models which incubate the conceptual innovations crucial to the ongoing development of scientific research programs. In the remainder of this paper, I hope to make 
this claim plausible in the case of chemical structure. I begin by characterizing Pierre Duhem's understanding of structural formulas. Duhem's position is interesting because it reflects his more general approach to physical theory, which was criticized by Mary Hesse in one of the seminal works arguing for the essential role of models in science. Hesse's core insight, and some of her useful terminology, are then used to suggest that Duhem's conception of structural formulas is impoverished precisely because it neglects their role as models. I will support this conclusion by briefly elucidating the dynamic role of visual representations of structure in the development of conformational analysis, primarily in the work of D. H. R. Barton and Odd Hassel, co-winners of the 1969 Nobel Prize in Chemistry.

\section{Duhem, Hesse, and Visual Representations of Chemical Structure}

The history of visual representations of chemical structure is a long and complicated one. ${ }^{1}$ Compositional formulas, which reflect the relative amounts of the various elements in chemical compounds, eventually gave way to structural formulas, which distinguish isomers in terms of connectivity. Structural formulas were in turn modified in order to make way for stereochemistry, where optical isomers are distinguished by their three-dimensional arrangements in space (configuration). By the late nineteenth century, many chemists seem to have interpreted these structural formulas as at least partially realistic models of the entities responsible for the chemical processes that they create and measure in the laboratory. Others, such as Pierre Duhem-perhaps because so little was known about either the structure of atoms or the nature of chemical bonding-resisted this interpretation. For Duhem, the goal of the development of structural formulas was purely notational: "the construction of schemas appropriate to represent truths of the experimental order" which could and should be done, he thought, while having "attributed to these schemas no relationship with the constitution of matter itself" (Duhem 2002, 284).

Duhem's understanding of structural formulas seems to limit the significance of such formulas to being "symbols appropriate for representing and classifying the facts" $(2002,285)$ and he thereby denies their role as models of chemical compounds. Duhem plausibly relates how the notation system of the chemists of his time encodes the experimental facts, such as isomer counting experiments, which were actually its empirical foundation. When frozen in time by Duhem, the role of structural formulas as models is not obvious; they become merely a symbolic system that allows for a logical and unified organization of the experimental facts. He says:

The symbols employed in modern chemistry... are precise

1 For a readable introduction, see O.T. Benfey, From vital force to structural formulas, (Boston: Houghton Mifflin, 1964). 
instruments of classification and discovery as long as they are regarded only as the elements of a language, of a notation, appropriately conveying to the eyes, under a particularly striking and precise form, the notion of analogous compounds, substances derived from one another, optical antipodes... when on the other hand, it is regarded as a reflection, as a sketch of the structure of a molecule, of the arrangement of atoms, of the shape of each of them, this leads to insoluble contradictions. (Duhem 2002, 88)

In chemistry, the notation system provides deductive systematization, which for Duhem is the hallmark of a well-developed physical theory. The alternative, as Needham (2002, xxvi) suggests, would be to suppose that "mere pictures" could be "the stuff of articulate theories."

The rather dismissive attitude that Duhem takes toward chemists who take their pictures too seriously is consistent with his more general attitude towards the role of models in science. For him, models and other imaginative aids may play a heuristic role in theory generation, but are ultimately an inessential distraction from the real business of science, which is deductive systematization. Whether or not this is a fair characterization of Duhem's position, it has been the target of some of the most important philosophical work devoted to establishing the essential role of models in science. Mary Hesse, following on the work of N. R. Campbell, criticizes Duhem's understanding of physical theory precisely because it downplays the role of models in scientific theorizing. In particular, she alleges, scientific models play an essential role in the dynamic aspect of science because they allow it "to make predictions in new domains of phenomena" (Hesse 1966, 4).

To make her point, Hesse characterizes models as objects or systems of objects that are taken to be analogous to what would these days be called the target system. These models are merely analogous to, and not token representatives of, the target system because there are certain features of the model that are not intended to apply to the target system. Hesse refers to these properties as the negative analogy of the model. Similarly, there are features of the model that are explicitly intended to be carried over into the target system; these are the positive analogy. The crucial dynamic role of models in science, however, does not come from either of these sets of properties. Instead it is those "properties of the model about which we do not yet know whether they are positive or negative analogies," called the neutral analogy, which are the interesting properties because "they allow us to make new predictions" (Hesse 1966, 8). The difference between considering a target system using a merely formal system and using a model is that when employing the formal system we consider the target system only under the positive analogy, by attributing to it just those characteristics which we affirmatively take it to possess. When employing the model, the target system is considered not only under the positive 
analogy, but also in contrast to a set of properties it does not have, as well as in relation to an indefinite set of features that might be inferentially significant.

Hesse emphasizes the role of the neutral analogy in extending a scientific approach or theory by facilitating novel predictions. Novel predictions are on just about every philosopher's list of the desirable features of a successful theory or a progressive research program. Not all novel predictions are created equal, however. Hesse, for example, distinguishes between new relationships among the same types of evidence that were used to establish the theory in the first place and the relationships among, or with, new types of evidence. While the first sort of novel predictions are valuable, and may in some circumstances be sufficient to sustain a scientific approach, it is the second sort which really marks the expansion of a theory into new territory. Not surprisingly, Hesse finds that the essential role of models in science comes from their role in generating these conceptually innovative predictions. The basic idea is that when faced with the prospect of extending a theory into new territory, the properties of the neutral analogy supply good reasons internal to the model for expanding the approach in particular ways to accommodate new phenomena. Similarly, though Hesse herself does not develop this point, thinking about a target system in terms of a model may supply non-arbitrary reasons for modifying the model in particular ways in order to accommodate anomalous results. If the positive analogy generated predictions that are disconfirmed, then perhaps some aspects of this analogy must be dropped, or new distinctions might be imported from either the neutral or negative analogy. Alternatively, in what I think is an important omission from Hesse's account, the model itself might be changed, thereby modifying the candidate set of properties that must be sorted into the various analogies. The overall point is just that both conceptually novel predictions and the sorts of strategic adjustments that scientists must make in the face of unexpected results are constrained by their models.

I now want to return to visual representations of structure in chemistry and try to adapt some of Hesse's terminology and arguments to this particular case. If one holds, as Duhem seemed to, that structural formulas are merely elements of a symbolic notation system, then this is to treat them as bearing a purely formal relationship to the compounds they purport to denote. That is just to say that only the characteristics of the symbols explicitly related to particular experimental outcomes have potential significance, and this is all of the significance that they have. On the other hand, to treat a structural formula as a model is to regard it as "a sketch of the structure of a molecule." The elements of the symbolic system of chemists, so regarded, not only allow for the inference of particular experimental consequences, but also have some features in both the negative and neutral analogies. The differences between these two ways of regarding the elements of the symbolic systems of chemists can be expected to show up, if Hesse is right, in chemists' attempts to produce novel results 
within their structural research program or to adapt this program in the face of unexpected results. ${ }^{2}$ To evaluate the plausibility of her claims, it is necessary to consider cases where structural formulas were modified to accommodate conceptually novel results or generated conceptually novel predictions.

\section{Conformational Analysis and the Evolving Significance of Structure}

One of the most dramatic changes in the significance of chemists' visual representation of structure occurred during the middle third of the twentieth century with the gradual realization that the conformations of molecules (not just their configuration and connectivity) had a crucial role to play in understanding their physical and chemical behavior. A molecular conformation is, roughly, any of the three-dimensional arrangements of its constituent atoms in space resulting from rotations around single bonds. In broad outline, the development of conformational analysis is a story about how features of structural formulas that had originally been relegated to the negative and/or neutral analogy gradually were moved into the positive analogy. With new features available in the positive analogy, new concepts were crafted to organize the phenomena and then articulated throughout the domain. Though somewhat artificial, in order to relate the development of conformational analysis to the themes of this paper, it can be understood to have occurred in two phases. First, the prior understanding of structural formulas had to be found to be insufficient, and adjustments made to accommodate those insufficiencies. Second, once new features had been moved into the positive analogy, the consequences of that newly enhanced conception of structure had to be developed and articulated. These two phases correspond to the "back" and the "forth" of model dynamics: the first is the modification of models in response to experiment, and the second the generation of novel results using the modified models.

Using structural formulas to distinguish distinct chemical compounds required taking some features of those formulas to be significant, and others insignificant, to the individuation of chemical kinds. For example, double bonds were understood to restrict rotation, and so the distinct formulas possible by different orientations around these double bonds represented distinct compounds. On the other hand, the various physical models or structural formulas that could be generated by rotations about single bonds were distinct without representing different compounds. From a Duhemian point of view, one might say that these rotational variants were an incidental feature of the symbol

2 Interestingly, Duhem (2002) does acknowledge that part of what makes for a good "schema" is that it leads to discoveries. He is also clear that structural formulas have been fruitful in this sense, but the predictions he describes such formulas as generating are not what Hesse would call conceptually novel predictions. Instead they were the very same kinds of facts that led to the introduction of stereochemistry in the first place. 
system that needed to be ignored when deducing the experimental facts from the notation (like the font of the atomic symbols). By contrast, if one thinks of structural formulas as models, it would be more appropriate to say that these differences weren't important for the individuation of chemical compounds; this left it open whether these differences might be employed to explain other sorts of distinct experimental results.

Eventually, two new types of experimental results did force such modifications. The first was failed isomer counting experiments in which, beginning in 1922, chemists were able to distinguish optically active forms of compounds where, if all rotation about single bonds were free, there should not be any such forms. The second sort of evidence came from discrepancies between the observed and measured entropy of ethane. These discrepancies "could only be explained by a barrier to free rotation about the two methyl groups" (Barton 1969, 299). These new sorts of experimental results were accommodated by redistributing the features of models of structure among the positive, negative, and neutral analogy. Most fundamentally, the fact that a model has lots of rotational variants was no longer part of the negative analogy. It was an explanatorily significant fact that there are differences in the models resulting from rotations around single bonds. By imagining the atoms of a molecular model or structural formula to be interacting (either by attraction or repulsion) in a manner that varied according to the distance between them, the chemists looking to revise chemical structure could explain both why the rotations of ethane would be restricted and why there might be optically distinct forms of some strategically bulky organic molecules. The chemical structures of Duhem's time, when used as models, were carrying about with them a set of distinctions in the neutral analogy, ripe for the application of this idea.

Thinking in terms of such non-bonded interactions required interpreting chemists' representations of structure, including structural formulas, to be significant in new ways. However, perhaps because "there was no technique available to demonstrate the phenomenon experimentally" (Barton 1969, 299), this significance was not systematically explored until after the Second World War. Still, the rotational variants of structural formulas or physical models had demonstrated how useful they were by explaining several different sorts of novel experimental results (entropy measurements and failed isomer counting experiments) and had therefore earned their place in the positive analogy.

The integration of conformations into the mainstream practice of organic chemists began when Odd Hassel published his systematic investigations of the conformations of cyclohexane and its derivatives. Cyclohexane is an ideal experimental system for investigating the significance of conformations because, as investigation of a model will quickly show, there are only three conformations of this system that have no angle strain (what are now called the "chair," "boat," and "twist-boat"), out of the infinite number that 
are theoretically possible. Hassel's work showed how the conformational preferences of cyclohexane and its derivatives could be rationalized using repulsive non-bonding interactions in a way that had strong experimental support. Furthermore, he not only isolated a structural type in which the energetic implications of conformational differences were clear, but also developed structural concepts (what are now called axial and equatorial positions on the ring) useful in explaining the relative energies of structures of this type.

It was Barton who established the importance of conformational analysis in explaining and predicting the chemical behavior of synthetically important organic molecules. He did this by recognizing that steroids are instances of the structural type carefully studied by Hassel. So, just as with cyclohexane itself, the significance of conformations for the behavior of steroids can be understood by considering just a few of the infinitely many possible conformations. Better still, following Hassel, the axial and equatorial substituents in the steroid nucleus can be distinguished and their relative stability rationalized in terms of repulsive non-bonded interactions. Originally in 1950, Barton showed that "an enormous literature of stereochemical fact" $(1969,302)$ about steroids could be systematically and consistently interpreted using the conformational analysis of the steroid nucleus. This established by a sort of consilience of inductions that, at least in the case of steroid chemistry, conformations had an important role to play in understanding chemical behavior.

Between Hassel and Barton, not only had conformations proved themselves to be useful in explaining significant chemical behavior, but also a set of structural circumstances (and concepts) had been articulated that allowed chemists to clearly discern the implications of conformation. With these resources in place, chemists begin to apply these concepts in synthesis and experimental design. These cases of application depended, initially, on being able to recognize a set of structural circumstances in which conformational analysis is straightforward because it could be directly related back to prior successes. Of course, chemists were not content to apply conformational analysis just to cyclohexane and steroids; from this base, conformational analysis was articulated along several different avenues. It was applied to other molecules containing cyclohexane subunits, while quantitative approaches were developed and attempts were made to extrapolate the same basic approach used in analyzing cyclohexane to unsaturated six-membered rings and heterocyclic compounds. Barton ends his Nobel Prize Acceptance speech in 1969, with what now seems like a rather dramatic understatement, claiming that knowledge of the conformations of organic molecules "must soon have important consequences in biology" $(1969,309)$.

Once the rotational variants of structural formulas or molecular models were recognized to be significant, chemists still faced the daunting task of organizing 
and sorting these infinite structural variations into categories that could be inferentially connected with experimental results, and eventually lead to new experiments. This was not done in a top-down way, by somehow deducing the implications of non-bonded interactions for chemical reactions. Instead, this depended on finding a particular case where the conformational implications were clear and then generalizing and articulating from there. The concepts used to connect conformations with experiment came, initially, from considering cyclohexane. And models and visual representations of cyclohexane played a crucial role in both the recognition of these concepts and their connection to experiment. Chemists focused on cyclohexane because they already knew, from the manipulation of models, that it had just a small number of strain-free conformations. Examination of these conformations led to the important distinction between axial and equatorial positions about the ring, which was subsequently linked with important energy differences between structural variants of cyclohexane (including, ultimately, steroids). These conformational features of cyclohexane are not features shared by most molecules, but they turned out to be crucial in elucidating the chemical consequences of conformations. The distinctions between conformations that were actually used in order to connect this new aspect of chemical structure with experiment were available only in concrete representations of a particular structure. Models of cyclohexane are rich in discernible differences not previously identified as significant in chemical explanations. These previously-neutral features supplied the concepts that eventually got connected with experimental results. It was then by generalizing, adapting, and articulating these foothold concepts that the broad applicability and novel applications of conformations were developed.

\section{Conclusion}

I hope to have brought out two distinct ways that visual representations of structure contributed to the evolution of the research program of structural chemistry in their capacity as models. In the first case, models of structure supplied a rich array of rotational variants that had not previously been recognized to be inferentially significant. When supplemented with the idea of non-bonded interactions, these features of the model could be used to explain anomalous results. Chemists did not abandon the structural research program when they found that, contrary to earlier expectations, optically distinct substances could be isolated without any distinct structural formula to correspond to them. Instead, they modified their models of structure, taking features readily available in the model and attributing new inferential significance to them. General features carried around in the models were appropriated in order to modify the conception of chemical structure in the face of new experimental results.

In the second case, it was particular models of structure that supplied the 
foothold concepts originally connecting conformations with experiment. Visual or material representations of particular molecules have many specific features that can be explored for their potential inferential significance. In this sense, models of particulars are cognitively richer than general types of models or abstract theories. They have all sorts of features that might turn out to support important inferences about the target system. This extra cognitive content seems to have been crucial to developing conformational analysis. The experimental significance of conformations was not deduced from some general theory of non-boded interactions. Instead, chemists isolated particular cases where this significance was clear, used very local concepts to explain and predict in those cases, and then generalized from there. In this case, then, particular features carried around in the model were appropriated to develop and articulate the experimental consequences of chemical conformations.

At least in general terms, Hesse seems to be correct. Models, and in this case visual representations, do play an important role in the dynamics of science by supplying concepts or features that can be appropriated to modify or develop a research program. It was (and is) important that chemists understand their structural formulas, contra Duhem, to be "sketches of the structure of a molecule." Visual representations of structure, and models of structure more generally, act as incubators for the concepts essential to modifying and teasing out the experimental consequences of chemical structure.

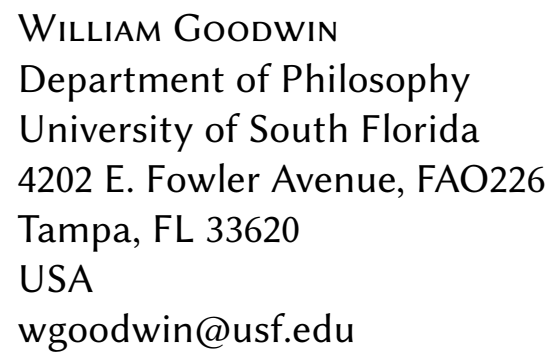

\section{RefERENCES}

Barton, Derek. H. R. 1969. The principles of conformational analysis. Nobel Lecture, December 11, 1969.

[www.nobelprize.org/nobel_prizes/chemistry/laureates/1969/barton-lecture.html]

Conant, James. 1947. On Understanding Science: An Historical Approach. New Haven, CT: Yale University Press.

Duhem, Pierre. 2002. Mixture and Chemical Combination. ed. and trans. Paul Needham. Dordrecht, The Netherlands: Kluwer Academic Publishers.

Goodwin, William. 2008. Structural formulas and explanation in organic chemistry. Foundations of Chemistry 10(2): 117-27. 
Goodwin, William. 2009. Visual representations in science. Philosophy of Science 76(3): 372-90.

Hesse, Mary. 1966. Models and Analogies in Science. South Bend, IN: University of Notre Dame Press.

Needham, Paul. 2002. Introduction. In Mixture and Chemical Combination ed. and trans. Paul Needham. Dordrecht, The Netherlands: Kluwer Academic Publishers. 